\title{
Evaluación psicométrica de una escala de autoeficacia de la conducta alimentaria
}

\author{
A psychometric assessment of a \\ self- efficacy eating consumption scale
}

\begin{abstract}
Background: We evaluated the reliability, factorial validity and criteria validity of a psychometric test designed to measure eating behavior self- efficacy. Materials and methods: This construct was measured using a 21 item scale appplied to a sample of 300 between 14 and 74 years $(M=29.89)$, from the Metropolitan Area of México City, Mexico. Results: Data analysis revealed a four factor structure with high reliability. These factors characterized 4 different types of eating behaviors: 1) high fat eating consumption; 2) healthy eating consumption; 3) sweets consumption and 4) drink consumption. Additionally, we measured physical activity as a factor that could be related with healthy eating. Conclusion. We analyzed feasibility of using this psychometric tool to assess self-efficacy in our sample and its potential implication for nutritional programs. It is also considered feasible to apply this scale in new settings, such as educational programs on nutrition and in the nutritionist practice, since it can provide a predictive tool for eating behaviors.

Key words: Eating self- efficacy; scale; factorial validity; reliability and eating behavior.
\end{abstract}

\section{INTRODUCCIÓN}

La teoría del aprendizaje social o social cognitiva (1-3), incluye un constructo teórico que se utiliza frecuentemente dentro de los modelos de cambio conductual nombrado autoeficacia, que se define como la capacidad personal del individuo de controlar su propia conducta cognitivamente, la convicción que se tiene (o no) sobre la viabilidad de que la conducta que se va a ejecutar logrará expresarse exitosamente en la situación o contexto que ha sido requerida. Esto le permite al individuo actuar o producir un resultado eficiente en una situación y/o contexto determinado (4).

La noción de autoeficacia $(1,2,4)$ señala que las percepciones de eficacia varían de modo significativo entre las distintas situaciones y tareas que se llevan a cabo ordinariamente. Las personas que se sienten o perciben así mismos como eficaces, poseen metas más altas y persisten más en sus propósitos que aquellos que tienen un bajo control personal ante ciertos retos o situaciones $(1,4)$.
Jorge Palacios (1)

Víctor Ramírez (2)

Miriam Anaya (3)

Hebert Luis Hernández (4)

Rubén Martínez (5)

(1) Maestría en Nutrición Humana, Maestría en Neurometabolismo, Clínica del Sistema Nervioso, Facultad de Ciencias Naturales, Universidad Autónoma de Querétaro, México, (2) Instituto de Investigación Médica Mercedes y Martín Ferreyra INIMEC-CONICET-UNC
Córdoba Argentina.

(3) Maestría en Nutrición Humana, Facultad de Ciencias Naturales, Universidad Autónoma de Querétaro, México. (4) Maestría en Neurometabolismo, Clínica del Sistema Nervioso y Laboratorio Multidisciplinario de Investigación Biomédica Avanzada, Facultad de Medicina, Universidad Autónoma de Querétaro, México. (5) Maestría en Neurometabolismo, Clínica del Sistema Nervioso y Laboratorio Multidisciplinario de Investigación Biomédica Avanzada, Facultad de Psicología, Universidad Autónoma de Querétaro, México.

Dirigir correspondencia a: Jorge Raúl Palacios Delgado Facultad de Ciencias Naturales, Universidad Autónoma de Querétaro Cerro de las Campanas S/N, Santiago de Querétaro, México Teléfono: (01) 4422078517 Email: drjpalacios81@gmail.com

Este trabajo fue recibido el 25 de Mayo de 2016 y aceptado para ser publicado el 6 de Diciembre de 2016.

Los pensamientos y acciones que integran la autoeficacia son precursores inmediatos y específicos de la conducta (5). De esta manera la autoeficacia puede considerarse como un determinante de la conducta $(5,6)$. Está hipótesis se ha puesto a prueba mediante la predicción de distintos comportamientos (7-13).

Algunos autores $(2,3,5,14)$ señalan que la capacidad de aprendizaje que el individuo muestra durante la adquisición de nuevas conductas depende del grado de eficacia con la que cuenta el individuo, para este tipo de conductas y/o cogniciones.

En el presente trabajo nos interesa evaluar la autoeficacia en relación a conductas alimentarias consideradas saludables. De esta manera, diseñar un instrumento que mida si las personas tienen o no un alto sentido de autoeficacia de la conducta alimentaria, debería estar acompañado de una mayor capacidad de realizar comportamientos alimentarios saludables por parte del individuo. 
Dentro de la salud (15) la autoeficacia cumple un rol importante en la realización de una gran variedad de conductas saludables, como son la reducción de peso (16-19), características de la conducta alimentaria (20) la alimentación baja en calorías o la reducción del consumo calórico $(21,22)$. Por ejemplo, la autoeficacia modera la ingesta de alimentos saludables en adolescentes (23). Gutiérrez, Lippke, Renner, Kwon y Schwarzer (24), mencionan que la autoeficacia es un factor relevante en el proceso de cambio de conductas saludables, al predecir en este caso la adherencia a una dieta baja en calorías en mujeres.

Se ha estudiado la asociación entre la autoeficacia y la actividad física $(25,26)$, encontrando que quienes tienen una alta autoeficacia son efectivamente más activos en su actividad física. Adicionalmente se ha encontrado que la autoeficacia es una variable importante para predecir la adopción o adherencia y mantenimiento a un régimen del ejercicio físico (27).

En pacientes con esclerosis múltiple, la autoeficacia contribuye a la realización de actividad física (28), también en pacientes en procesos de rehabilitación por problemas cardíacos. La autoeficacia se considera un factor facilitador del ejercicio físico (29) y en la rehabilitación ortopédica es un predictor efectivo para la adherencia del ejercicio físico entre 4 y 12 meses después de la intervención (30).

El constructo de autoeficacia se ha evaluado en conductas saludables, e incluso existen diversos instrumentos que a nivel internacional miden el control y manejo del peso $(31,32)$ la experiencia de alimentación (33) el control alimenticio (34) la alimentación saludable y el manejo del peso (35), la conducta alimentaria y la ingesta $(36,37)$, los escenarios alimenticios y situaciones de dieta (38), las situaciones de ingesta de alimentos (39) y circunstancias alimenticias de dieta (40). En México, se han realizado algunos estudios para medir la autoeficacia a través de escalas que evalúan la actividad física (41), el control de peso $(42-44)$ o conductas saludables $(45,46)$.

Aunque se han reportado varios instrumentos de evaluación de autoeficacia de la conducta alimenticia en varios lugares del mundo $(32,35,37,43,44-46)$, no hay estudios en población Mexicana que puedan utilizarse con el propósito de medir el constructo de autoeficacia adecuadamente, lo que debe de hacerse exprofeso ya que los instrumentos elaborados para medir este constructo han de contener los alimentos disponibles en nuestro territorio. Debemos incluir en la muestra personas de diferentes edades que componen a nuestra población, además de incorporar un análisis de los alimentos saludables, alimentos con alto contenido calórico y todo tipo de bebidas, con particular énfasis en las bebidas endulzadas con saborizantes naturales o artificiales.

Lo anterior es relevante si consideramos que en nuestro país el consumo de bebidas endulzadas es elevado y se ha considerado como factor de riesgo para la salud (47) ya que hay evidencias que este hábito de consumo propicia sobrepeso, obesidad y alteraciones metabólicas $(48,49)$, aumentando la probabilidad de diabetes tipo 2 (50) y un incremento en el riesgo cardiovascular. Todos estos problemas de salud presentan porcentajes alarmantes en nuestro país como lo demuestra la más reciente Encuesta Nacional de Salud (47) indicando que en adolescentes, el 35\% presentan sobrepeso u obesidad, pero para los adultos se estima que la proporción de individuos con indicadores de sobrepeso u obesidad está alrededor del 70\% para hombres y mujeres.

Los estudios previos que miden autoeficacia sobre conductas saludables, se agrupan en tres categorías: A) Medidas sobre la actividad física; B) Control y mantenimiento del peso y C) Alimentación, que integra: 1) las situaciones de ingesta de alimentos y 2) la conducta de ingesta alimenticia.

Al respecto de ésta última categoría, aunque existen algunos instrumentos que miden la autoeficacia de la conducta alimentaria, el principal problema que tienen es que no permiten discriminar entre conducta alimenticia y actividad física, ya que suelen combinarlos en un mismo factor o bien en un solo instrumento para el control de peso $(45,46)$. Otros instrumentos poseen dificultades en la medición y validez del constructo, tanto en su dimensionalidad, como en la estructura factorial o tienen deficiencias en el formato de respuesta; por esta razón integramos un nuevo instrumento de autoeficacia de la conducta alimentaria, ya que es uno de los principales determinantes del comportamiento saludable.

En términos de validez, se pretende estimar en el instrumento tres aspectos distinguibles (51-53): 1) el contenido; 2) el factorial y 3) el de criterio (predictiva). Es importante considerar que al pretender evaluar constructos universales (Etic) en características idiosincráticas particulares de cada cultura (Emic), es necesario identificar los indicadores del constructo que sean relevantes, adecuados y sensibles a la cultura en la que se van a medir y operacionalizar $(10,54-57)$. Este principio es central para determinar las características psicométricas del presente instrumento.

La integración de una medida de autoeficacia alimentaria que incorpore una o más categorías, válida, confiable y sensible a la cultura mexicana podría ser utilizada para fines diagnósticos, de intervención o de investigación que permita obtener información de la capacidad de las personas para manejar adecuadamente conductas saludables y de alimentación, además de estar ligada al propósito de realizar programas de educación nutricional. Es tal la capacidad predictiva que potencialmente tiene la métrica de autoeficacia, que se hace importante tenerla disponible para la conducta alimentaria como una herramienta que puede ser útil a nutriólogos, médicos y psicólogos.

El instrumento integrado facilitará el estudio la autoeficacia de la conducta alimentaria en relación con problemas de obesidad o sobrepeso de nuestro país. Puede convertirse en una herramienta aplicable en la clínica, como herramienta diagnóstica y para el seguimiento de estrategias terapéuticas. Además, al considerar este constructo como parte de la teoría del aprendizaje social o social cognitiva, la autoeficacia tiene un papel fundamental en los modelos de cambio de conducta $(1,3,4,12,58,59)$.

Considerando el uso potencial de las escalas de autoeficacia vinculadas a conductas saludables, el objetivo del presente estudio fue integrar una escala sobre la autoeficacia de la conducta alimentaria y estimar sus propiedades psicométricas (confiabilidad, validez factorial y de criterio).

\section{SUJETOS Y MÉTODOS \\ Participantes}

Se seleccionó una muestra no probabilística de tipo intencional de 300 personas, 134 hombres y 166 mujeres, con un rango de edad entre 14 y 74 años y una media de 29,89 años (DE $=13,5)$, de la Zona Metropolitana de la Ciudad de México. El 60,7\% indicó no realizar alguna actividad física o practicar algún deporte, mientras que sólo 39,3\% declaró realizar algún tipo de actividad física.

Instrumento

Definimos la autoeficacia ante el consumo alimenticio como la capacidad percibida que tienen las personas para 
disminuir el consumo de alimentos altos en calorías, evitar alimentos dulces y comer alimentos saludables $(3,4)$. Para evaluar este constructo se integró un instrumento partiendo de los lineamientos propuestos por Bandura6 y considerando las sugerencias en la elaboración de instrumentos de algunos autores (56-60), así como de ítems similares de otras escalas que miden autoeficacia $(7,10,13,42,46)$. Los ítems se redactaron considerando que su contenido fuera congruente con la teoría social cognitiva $(2-4,58,59)$ utilizada en el presente estudio.

El instrumento final estuvo conformado por 21 ítems con opción de respuesta que va de 1 a 10, donde 1 representa la ausencia de capacidad y 10 ser muy capaz en disminuir la ingesta de productos calóricos o dulces y aumentar el consumo de alimentos saludables. Adicionalmente se les preguntó si realizaban algún tipo de actividad física.

\section{Procedimiento}

Se aplicó el instrumento de forma individual a las personas en su propio domicilio, en plazas y lugares públicos o bien utilizando a grupos escolares para tal fin en un tiempo aproximado de 15 minutos. Se les explicó que el cuestionario era para conocer algunas actividades relacionadas con su alimentación.

\section{Consideraciones éticas}

A todos los participantes se les aclaró que la información era anónima, se les garantizó la confidencialidad de los datos proporcionados y se resolvieron las dudas que tuvieran. Se utilizó el consentimiento informado de los participantes y de las autoridades escolares. El protocolo de investigación fue establecido de acuerdo al Reglamento de la Ley General de Salud, en su apartado sobre investigación en seres humanos (61).

\section{Análisis de datos}

Los análisis de datos se realizaron considerando las variables utilizadas y fueron capturados y analizados con el programa SPSS 14. Para evaluar las características psicométricas del instrumento, se utilizaron diferentes análisis estadísticos. Se utilizó la frecuencia de cada ítem para conocer su distribución. Los cuartiles y la prueba t de Student permitió comparar la puntuación total del instrumento para identificar aquellos ítems que no discriminaban del total de la escala. Se realizó un análisis factorial exploratorio para obtener la validez del constructo. Se utilizaron dos procedimientos (el método de dos mitades y el Alfa de Cronbach) para valorar la confiabilidad del instrumento. Se realizaron correlaciones entre factores de la escala para estimar la validez convergente. Se utilizaron medidas de tendencia central (media y desviación estándar) para obtener las puntuaciones de la escala utilizada y por último se llevó a cabo una regresión logística para estimar la validez referida a un criterio.

\section{RESULTADOS}

Se revisó la frecuencia de cada uno de los ítems presentados a los participantes y con ello se evaluó su distribución, lo que permitió distinguir diferentes grupos utilizando los cuartiles. Mediante el uso de la prueba t de Student, se comparó al grupo alto (arriba del tercer cuartil) y bajo (debajo del primer cuartil) en la puntuación total del instrumento, para obtener el análisis de discriminación de reactivos, para este caso se consideró que un ítem discriminaba cuando obtuvimos una diferencia significativa con una probabilidad $\leq 0,05$. Para seleccionar los reactivos que mejor discriminaron, se utilizó el criterio de correlación ítem-total, eliminando aquellos ítems que obtuvieran una correlación menor a 0,20.

Para obtener la validez de constructo de la escala, se realizó un análisis factorial exploratorio de ejes principales con rotación ortogonal, para obtener la agrupación de los ítems. Como requisito previo al análisis factorial se evaluó la adecuación de las matrices de correlaciones utilizando el índice de adecuación de la muestra de Kaiser-Meyer-Olkin (KMO) obteniendo un valor de 0,928 considerado como excelente.

El Índice de Esfericidad de Bartlett's fue significativo $(X 2=3342,92 ; d f=210 ; p<0,001)$ indicando la presencia de correlaciones significativas entre los ítems y que la multidimensionalidad es adecuada, por lo tanto, permitió llevar a cabo el análisis factorial. El análisis factorial exploratorio mostró 4 factores: 1) alimentos con alto contenido en grasas; 2) alimentos que contienen frutas y verduras; 3 ) alimentos que contienen dulce y 4) bebidas. El criterio para seleccionar un factor fue el punto de quiebre del gráfico de sedimentación de Catell; además se eligió que el valor propio fuera superior a 1,0. Para interpretar los factores, el criterio de inclusión para un ítem dentro de cada factor fue que presentara un peso factorial mayor a 0,30 y no tuviera un peso factorial similar en otro factor (61). Adicionalmente se consideró la claridad conceptual de cada dimensión a partir de lo encontrado en estudios similares, y que cada factor contará como mínimo con tres ítems. La solución factorial obtenida explica 54,3\% de la varianza total, considerado como suficiente e indicativo de que se ha obtenido la cantidad necesaria para representar el constructo medido. La tabla 1 muestra los ítems agrupados por factores y sus respectivas saturaciones.

La confiabilidad total del instrumento se evaluó por dos procedimientos diferentes. El primero fue el método de dos mitades (52). La parte 1 con 11 afirmaciones obtuvo un valor de 0,903 y la parte dos con 10 aseveraciones mostró un valor de 0,864 ; la correlación entre formas fue de 0,792; con un coeficiente Spearman-Brown de longitud desigual de 0,884 y para el modelo de Guttman una fiabilidad de 0,875. El segundo procedimiento para obtener la confiabilidad del instrumento fue por medio del análisis de consistencia interna (coeficiente Alpha de (ronbach) para el instrumento completo, el cual mostró un índice de 0,93 (IC95\%=0,92-0,94). Los niveles de confiabilidad obtenidos indican que los ítems del instrumento son homogéneos en la medición del constructo, así como la pertinencia de señalar que la escala puede evaluarse mediante la relación existe entre todos los ítems que la conforman.

El análisis descriptivo obtenido para cada factor se encuentra en la tabla 2. Los valores promedio se encuentran por sobre la media teórica, indicando los niveles en autoeficacia en las respuestas de las personas. Las desviaciones estándar cercanas a dos puntos, son relativamente consistentes a través de los factores. El intervalo de confianza de la fiabilidad no disminuye de 0,70 y no supera el 0,90, indicando la estabilidad de la escala.

Se realizaron correlaciones multivariadas de Pearson entre los cuatro factores obtenidos en la escala. La tabla 3 muestra que los cuatro factores correlacionan de forma positiva y significativa. La capacidad para evitar alimentos altos en grasa correlaciona con los alimentos dulces, la autoeficacia para consumir alimentos saludables y en último término con la autoeficacia ante las bebidas. El factor de alimentos saludables correlaciona con la autoeficacia ante alimentos altos en grasas y con la autoeficacia ante alimentos dulces, en la misma dirección e intensidad y en menor magnitud con la autoeficacia para consumir bebidas endulzadas. El factor de autoeficacia ante bebidas endulzadas, se relacionó en primer 
lugar con la capacidad para alimentase sanamente, seguido de los productos dulces y con la capacidad de rechazar los alimentos altos en grasa. Los niveles de correlación más altos se encontraron entre los factores de alimentos altos en grasa, así como con los alimentos dulces, lo que permite asumir que son variables cercanas y posibles factores involucrados en el sobrepeso y la obesidad en nuestro país.

Para obtener la validez referida a un criterio mediante una regresión logística, se estimó la proporción de cada subescala de la autoeficacia del consumo alimenticio entre quienes realizan actividad física o algún deporte y los que no lo realizan. Los resultados mostraron que las características de la autoeficacia que se estimó en mayor proporción para los que realizan actividad física o deportiva fue el consumo de alimentos sanos (OR=1,3; IC 95\%=1,0-2,6; $p=0,005)$, con una pseudo $\mathrm{R}^{2}$ de Negelkerke $=0,049$ y un porcentaje correcto de clasificación de 60,7\%.

\section{DISCUSIÓN}

Los resultados obtenidos para la escala analizada proveen apoyo a la validez de la medida de autoeficacia alimentaria a través de cuatro subescalas válidas y confiables con una extensión relativamente pequeña, lo que facilita que sea empleada junto a otros instrumentos que examinen diversos aspectos del comportamiento alimentario. Los ítems fueron elaborados para medir la autoeficacia alimentaria a partir de la ingesta de alimentos que las personas pueden Ilevar acabo. Los rubros que cubre el instrumento son: la autoeficacia para disminuir el consumo de alimentos altos en grasa, capacidad para elegir alimentos saludables, disminuir el consumo de alimentos

TABLA 1

Análisis factorial de la escala de autoeficacia del consumo alimenticio. México, 2014.

\section{Ítems}

Puedo dejar de comer alimentos chatarra (frituras, pasteles, galletas, papas), aunque tenga hambre

Puedo dejar de comer frituras o papas

Soy capaz de comer menos alimentos fritos o con mucha grasa

Soy capaz de rechazar comida chatarra cuando alguien me la ofrece

Soy capaz de rechazar invitaciones a comer alimentos ricos en grasas y carbohidratos como quesadillas, tacos, tortas o tamales

Soy capaz de comer frutas y verduras diariamente

Soy capaz de comer ensaladas cuatro veces por semana

Soy capaz de elegir una fruta en lugar de un pastelito como postre

0,616

Soy capaz de comer verduras frente a personas que están comiendo alimentos dulces

Puedo comer alimentos saludables cuando estoy con mis amigas (os)

Puedo evitar comer dulces o chocolates cada vez que se me antojen

Soy capaz de dejar de comer barras energéticas y comer una fruta

0,530

Soy capaz de disminuir mi consumo de cereal

(p.e. Corn flakes, Zucaritas, etc.)

Soy capaz de dejar de comer pastelitos

Puedo evitar comer frutas en almíbar o procesadas (Duraznos, Piña, etc.) aunque se me antojen

Soy capaz de dejar de comer mermelada, crema de cacao o cajeta

Soy capaz de eliminar de mi dieta dulces y chocolates

Puedo dejar de comer dulces o chocolates cuando me siento nervioso (a)

0,309

Puedo dejar de tomar refresco y beber agua natural

Puedo dejar de tomar jugos embotellados o agua de sobre y

beber agua natural.

Puedo dejar de tomar agua con saborizantes (p.e. Tang, Zuko, etc.) y tomar agua de frutas naturales.

Varianza explicada
0,330 $18,8 \%$
$14,6 \%$

$13,8 \%$ 
dulces y el cambio de bebidas endulzadas, que constituyen cuatro aspectos que se han reportado en la literatura como característicos del consumo alimenticio $(31,35,37,43,44,46$, 49). La obtención de estas dimensiones aporta evidencia a la integración de la teoría social cognoscitiva y la autoeficacia (1, $3,4,30)$, al vincularla con comportamientos saludables (11, $21,24,31)$ lo que permite aumentar la evidencia empírica existente respecto a que la autoeficacia puede ser aplicada para comprender y explicar el rol que tiene en los patrones alimenticios. Además la autoeficacia alimentaria con los cuatro factores aquí encontrados, permite distinguir en la muestra de mexicanos la posible adopción de hábitos de alimentación que afectan su salud, así como la capacidad de realizar y mantener comportamientos alimenticios sanos al ejecutar acciones para tener un estilo de vida saludable.

Al comparar la escala obtenida en este estudio con otras que intentaron medir el consumo de alimentos, se encuentran algunas coincidencias en el número de factores encontrados en varios estudios $(31,37,39,42-44,46)$, aunque en otros obtienen solo dos factores $(33,35,36)$. Las discrepancias con dichos estudios puede deberse a que en algunos casos (33, 36), evalúan la autoeficacia ante situaciones de alimentación, en lugar de la alimentación en sí misma; en el caso de WilsonBarlow y cols., (35) la diferencia puede encontrarse en que miden además de la alimentación saludable, el control del peso como su segundo factor. Román y cols., (32) encontró un factor nombrado estilos alimenticios y externalidad, que conjunta afirmaciones de los factores de alimentos altos en grasa y alimentos dulces mostrado en el presente estudio. Sallis y cols., (37) halló factores como la reducción de alimentos calóricos y alimentos grasos, coincidentes con los factores mostrados en nuestros resultados. Las dimensiones que incorporan algunos de los autores Mexicanos $(42-44,46)$, se refieren a estilos alimenticios no saludables, en correspondencia a los factores de consumo de alimentos dulces y consumo de alimentos altos en grasa, lo cuales conseguimos medir en la presente inves- tigación. El factor obtenido en el presente estudio sobre los alimentos saludables confirma lo encontrado previamente (21, $22,32,35)$ sobre una alimentación saludable. Por último, los factores que obtuvimos en nuestra escala, particularmente el factor de consumo de bebidas, difiere claramente de medidas previas de autoeficacia. En estudios previos de autoeficacia alimentaria se han medido factores que incluyen algún tipo de bebidas y nuestros resultados apoyan lo encontrado previamente $(20,49)$.

Para estimar el grado en que el instrumento que se integró mide realmente autoeficacia alimentaria, se evaluaron tres aspectos de su validez (51-53). En primer lugar, la validez de contenido se conformó por categorías alimenticias (grasas, carbohidratos, frutas, verduras y bebidas), como se muestra en los resultados. Consideramos que la medición de la autoeficacia relacionada con la conducta alimentaria puede implicar distintas categorías que pueden ser agrupadas en distintos factores como se ha encontrado previamente. Estas pueden incluir situaciones o contextos que promueven el consumo de alimentos $(31,38-40)$ o conductas particulares de ingesta de alimentos (32-37). En segundo lugar, la validez factorial de constructo, se logró por medio de la integración de cuatro factores con diferentes comportamientos del consumo alimentario y su posterior correlación inter-escalas, lo que sugiere que la autoeficacia que se tiene sobre una conducta alimentaria tiende a estar vinculada con la autoeficacia de otras conductas alimentarias, es decir, los individuos que puedan disminuir el consumo de alimentos dulces, tienen la capacidad de dejar de comer alimentos con grasa, así mismo, las personas que son capaces de realizar una reducción de la conducta de ingesta de alimentos poco sanos (menos alimentos fritos o con mucha grasa), tienen también la capacidad de realizar otros comportamientos para cuidar su alimentación (comer frutas y verduras diariamente). Además quien manifiesta ser competente en mantener una alimentación sana, tomará alguna bebida saludable (dejar de tomar refresco y beber agua natural), de la

\section{TABLA 2}

Estadística descriptiva de la escala de autoeficacia alimenticia. México, 2014.

\begin{tabular}{lcccccc}
\hline & $M$ & $D E$ & Ítems & Rango & a & IC 95\% \\
Alimentos altos en grasa & 7,19 & 2,0 & 5 & $1-10$ & 0,85 & $0,82-0,88$ \\
Alimentación saludable & 7,96 & 1,8 & 5 & $1-10$ & 0,84 & $0,81-0,87$ \\
Alimentos dulces & 8,03 & 1,6 & 6 & $1-10$ & 0,79 & $0,76-0,83$ \\
Bebidas & 7,89 & 2,1 & 3 & $1-10$ & 0,75 & $0,70-0,80$ \\
\hline
\end{tabular}

\section{TABLA 3}

Correlaciones interescala de la autoeficacia del consumo alimenticio. México, 2014.

\begin{tabular}{|c|c|c|c|c|}
\hline & Grasas & Saludable & Dulces & Bebidas \\
\hline Alimentos altos en grasa & -- & $0,616^{* *}$ & $0,695^{* *}$ & $0,564^{* *}$ \\
\hline Alimentación saludable & & -- & $0,616^{* *}$ & $0,606^{* *}$ \\
\hline Alimentos dulces & & & -- & $0,598^{* *}$ \\
\hline Bebidas & & & & -- \\
\hline
\end{tabular}


misma forma, quién mantiene el consumo de bebidas sanas, disminuye la ingesta de alimentos altos en grasa y alimentos endulzados. Examinar el contenido de cada subescala, permite utilizar cada factor por separado o como un instrumento integrado al evaluar tanto el consumo de alimentos, como la capacidad cognitiva para realizarlo.

La obtención de una medición que incorpore cuatro factores del comportamiento alimentario, se apoya en la idea de algunos autores $(21,32,34,35,37,42-44,46)$ que sugieren que la autoeficacia alimenticia puede implicar distintas conductas, como evitar comer carne o alimentos fritos, comer ensaladas en la cena, consumir frutas y vegetales, resistir comer galletas o alimentos dulces. Se encontraron diferencias con autores $(16,40,45)$ que evalúan la autoeficacia con pocos ítems, referentes a conductas generales en salud o que se encuentran encaminados a evaluar situaciones que favorecen el consumo $(31,33,36,38,39)$. Estas diferencias parecen indicar que en esta muestra de mexicanos es posible abordar la autoeficacia alimentaria por medio de dimensiones que incluyan varias acciones del consumo de alimentos, reafirmando que la autoeficacia vinculada a la ingesta de alimentos y bebidas puede medirse por una serie de ítems contenidos por cuatro factores que le otorgan congruencia conceptual para obtener validez factorial de los ítems tal y como han sido evaluados en el presente estudio.

Para determinar la validez predictiva o referida a un criterio de la escala propuesta en éste estudio, se utilizó como criterio la realización de actividad física o deportiva como lo proponen otros estudios $(25,26,29,41)$. Los datos encontrados sugieren que la autoeficacia sobre la conducta alimentaria saludable se encuentra presente en las personas que realizan algún tipo de actividad física o deportiva de manera que este hallazgo apoya lo encontrado por otros estudios (16-19, 21, $31,32,35,41,42-44,46)$ al argumentar que para el control de peso se debe combinar una alimentación saludable con regímenes de ejercicio o bien un incremento en la actividad física.

Los análisis relativos a la fiabilidad del instrumento son satisfactorios. La fiabilidad por mitades y por el método Guttman, muestra que para una población similar a la de la muestra estudiada, el instrumento es congruente, además de estable y que los resultados son similares a los reportados por estudios anteriores (31, 35, 37, 39, 42-44, 46). Los valores Alfa de Cronbach para el total del instrumento son indicativos de una adecuada consistencia interna entre los ítems que lo componen en su totalidad, así como para los valores de cada factor de manera independiente, otorgando soporte a la estructura factorial emergida del instrumento.

Los resultados que se presentan sobre el estimado psicométrico del instrumento, mostraron que la conceptuación de constructos universales (Etic) en este caso la autoeficacia y su medición por medio de su operacionalización vinculada en la conducta de alimentación en un contexto sociocultural particular (Emic), permite indicar que cuando se evalúan las propiedades psicométricas en términos de confiabilidad, validez y sensibilidad cultural de los instrumentos de medida, se debe de considerar las características etnopsicológicas de la población en donde se desarrollan $(10,54-57)$ para su posterior utilización en la clínica o la investigación.

Es importante señalar que el estudio presenta algunas limitaciones que hemos de tener en consideración. La primera corresponde al instrumento: se deberán revisar los reactivos de cada factor con la finalidad de incorporar una mayor variedad de comportamientos vinculados con la alimentación de la cultura mexicana; además de buscar aumentar los niveles de confiabilidad de las subescalas de alimentos dulces y bebidas, sobre todo si se pretende vincular la escala obtenida con variables como el índice de masa corporal, la actividad física y variables situacionales de la ingesta alimenticia. La segunda es referente al tamaño de la muestra, ya que es relativamente pequeña considerando la población que habita en la zona Metropolitana de la Ciudad de México, por lo que en futuros estudios se tendrá que ampliar la misma y tener en cuenta que estos resultados no se pueden generalizar a toda la población, sino que reflejan lo que está pasando con una muestra de ciudadanos mexicanos. Considerando el tamaño muestral obtenido parece conveniente que para corroborar los resultados logrados se deberá replicar el estudio en muestras más grandes de diferentes ciudades, considerando equilibrar el número de participantes por sexo y edad que responden a la escala.

La presente escala puede ser una herramienta eficaz para caracterizar a los mexicanos y sus hábitos de alimentación o bien para aquellos quienes ya ejecutan otro tipo de conductas alimenticias que pueden poner en riesgo su salud. Al poseer una comprensión más detallada del consumo alimenticio pueden desarrollarse estrategias más efectivas, enfocadas a promover los beneficios a la salud y a la calidad de vida de las personas; es decir, un estilo de vida más saludable.

De la información obtenida en el instrumento se derivan implicaciones prácticas debido a que la escala estimada puede ser útil para la evaluación de programas de educación nutricional, que pueden ser implementados desde ámbitos individuales, escolares, familiares, clínicos hasta sociales y comunitarios. Ante los problemas de sobrepeso y obesidad (47), uno de los modos para abordar esta problemática es mediante intervenciones realizadas para tal fin. Tales estrategias de educación alimenticia o nutricional deberán contemplar aspectos como los planteados en este estudio, para obtener efectos positivos en las intervenciones realizadas para regular la conducta alimenticia y mantener un peso adecuado. La educación nutricional debe ser una actividad permanente en la que se plantea la pertinencia de buscar un papel más activo en las personas para que promuevan y fomenten determinadas acciones vinculadas al cuidado de la alimentación.

De los resultados surgen ideas importantes que pueden ser consideradas en trabajos posteriores y como línea de investigación a futuro. Por un lado, el instrumento obtenido puede ser asociado con otras mediciones del control (control percibido, locus de control y autocontrol) alimenticio, los conocimientos o las actitudes alimenticias, las emociones que elicitan el consumo o bien incorporar a la personalidad como precursora de la autoeficacia alimenticia. En forma paralela, se considera integrar variables metabólicas o neurometabóicas, además de diferentes variables sociales a modelos que permitan explicar los problemas alimenticios, el sobrepeso y la obesidad de nuestro país.

El aporte de este estudio fue obtener distintos aspectos de validez para medir autoeficacia alimenticia con cuatro factores de una manera válida, fiable y culturalmente sensible para medir este tipo de autoeficacia en una muestra de individuos mexicanos. Un segundo aporte fue estimar empíricamente la validez de criterio de la escala con la finalidad de conocer la dirección, magnitud y proporción que tiene la autoeficacia alimenticia entre quienes realizan actividad física, con adecuados niveles de fiabilidad. El tercer aporte se sitúa en proporcionar a los investigadores, clínicos o terapeutas parámetros para poder ubicar la puntuación obtenida por un individuo en específico con un grupo de referencia en su capacidad para consumir o evitar diversos tipos de alimentos. 


\section{RESUMEN}

Objetivo: Evaluar la confiabilidad, la validez factorial y de criterio de un instrumento diseñado para medir autoeficacia alimentaria. Material y métodos: Se utilizó una escala de estimación de 21 ítems, aplicado a una muestra de 300 personas entre 14 y 74 años de edad $(M=29.89)$ de la Zona Metropolitana de la Ciudad de México, México. Resultados: El instrumento incorporó cuatro factores: 1) consumo de alimentos altos en grasa; 2) consumo de alimentos saludables; 3) consumo de alimentos dulces y 4) consumo de bebidas. Se encontró que la capacidad de alimentarse sanamente se encuentra presente en las personas que realizan actividad física frecuentemente. Conclusiones: Se plantea su utilidad en la evaluación de la autoeficacia de la conducta alimentaria en contextos como los programas de educación nutricional y la práctica del nutriólogo. Este instrumento puede proveer de una herramienta predictora de las conductas alimentarias.

Palabras clave: Autoeficacia alimenticia; escala; validez factorial; confiabilidad y psicología de la alimentación.

Conflicto de intereses: No existe conflictos de interés por parte de los autores que escriben este documento.

Agradecimientos: Proyecto apoyado a través de la Convocatoria de Estancia Posdoctoral Vinculada al Fortalecimiento de la Calidad del Posgrado Nacional de CONACYT. El segundo autor, agradece el apoyo otorgado por el FOFIUAQ, 2014.

\section{REFERENCIAS}

1. Bandura A. Self-efficacy: Toward a unifying theory of behavioral change. Psychol Rev. 1977; 84:191-215.

2. Bandura A. Social cognitive theory and exercise of control over HIV infection. In: DiClemente RJ, Peterson JL (Eds.). Preventing AIDS theories and methods of behavioral interventions. New York: Plenum Press, pp 89-116, 1994.

3. Bandura A. Social Cognitive Theory: An agentic perspective. Ann Rev Psychol. 2001; 52:1-26.

4. Bandura A. Self-efficacy: the exercise of control. New York: W.H. Freeman, pp 604, 1997.

5. Palacios DJ. Adolescent risk behaviors. México: Centro de Investigación e Innovación Biopsicosocial, AC, pp 189207,2011.

6. Bandura A. Guide for constructing self-efficacy scales. En: Pajares F, Urdan T, Eds. Self- efficacy, belief of adolescents USA: Age Publishing, pp 307-337, 2005.

7. Palacios DJ. Autoeficacia e intención conductual del consumo de tabaco en adolescentes: validez factorial y relación estructural. Adicciones. 2010; 22(4):325-30.

8. Palacios DJ, Parrao LM. Intention, skills and effectiveness to predict condom use. In: Rivera AS, Díaz-Loving $R$, Sánchez AR, Reyes LI. (Eds.). La Psicología Social en México. México: AMEPSO, pp 267-72, 2010.

9. Bandura A, Caprara GV, Barbanelli C, Gerbino M, Pastorelli $C$. Role of affective self- regulatory efficacy in diverse spheres of psychosocial functioning. Child Develop. 2003; 74(3):769-82.

10. Palacios DJ. Estimación psicométrica de la escala de autoeficacia ante conductas de riesgo para adolescentes en México. Psychol Interv. 2015; 1:1-7.

11. Palacios DJ, Bustos, AM. Análisis de la autoeficacia ambiental en el manejo de residuos sólidos, comparación en hombres y mujeres. Rev Psic Anáhuac. 2011; 14:31-8.

12. Palacios DJ, Bustos AM. Self-efficacy and environmental skills model as predictors of intention and pro-environ- mental disposition in youth. Rev Interc Psic Educ. 2012; 14(2);143-63.

13. Palacios DJ, Bustos AM. Validez factorial de la autoeficacia ambiental y su influencia estructural sobre la conducta proambiental en jóvenes. Rev Ibero Ev Psic. 2013; 35(1):95-111.

14. Bas M, Donmez S. Self-efficacy and restrained eating in relation to weight loss among overweight men and women in Turkey. Appetite. 2009; 52:209-16.

15. Villamarín F. Self-efficacy: Research in Health Psychology. Anuario Psic. 1994; 61:9-18.

16. Campos S, Pérez C. Self-efficacy and decisional conflict with the reduction of body weight in women. Rev Chil Nutr. 2007; 34(3):213-18.

17. Lugli RZ. Self-efficacy and locus of control: predictive variables of weight self-regulation in obese people. Pens Psic. 2011; 9(17):43-56.

18. Martin P, Dutton $G$, Brantley P. Self-Efficacy as a Predictor of Weight Change in African-American Women. Obes Res. 2004; 12(4):647-51.

19. Pinto B, Clark M, Cruess D, Szymanski S, Pera V. Changes in self-efficacy and decisional balance for exercise among obese women in a weight management program. Obes Res. 1999; 7(3):288-92.

20. Wardle J, Guthrie C, Sanderson S, Rapoport L. Development of the children's Eating behavior questionnaire. J Child Psychol Psych. 2001; 42(7):.963-70.

21. Luszczynska A, Tryburcy $M$, Schwarzer, $R$. Improving fruit and vegetable consumption: a self-efficacy intervention compared with a combined self-efficacy and planning intervention. Health Educ Res. 2007; 22(5):630-38.

22. Weller K, Greene G, Redding C, Paiva A, Lofgren I, Nash J. et al. Development and Validation of Green Eating Behaviors, Stage of Change, Decisional Balance, and SelfEfficacy Scales in College Students. J Nutr Educ Behav. 2014; 46(5):324-32.

23. Pearson $N$, Ball K, Crawford D. Predictors of changes in adolescents' consumption of fruits, vegetables and energydense snacks. Brit J Nutr. 2011; 105(5):795-803.

24. Gutiérrez DB, Lippke S, Renner B, Kwon S, Schwarzer R. Self-Efficacy and Planning Predict Dietary Behaviors in Costa Rican and South Korean Women: Two Moderated Mediation Analyses. Appl Psychol. 2009; 1:91-104.

25. Luszczynska A., Sheng CD, Mallach N, Pietron K, Mazurkiewicz M, Schwarzer R. Intentions, planning, and self-efficacy predict physical activity in Chinese and Polish adolescents: Two moderated mediation analyses. Int J Clin Health Psychol. 2010; 10(2):265-78.

26. Masse L, Heesch K, Eason K, Wilson M. Evaluating the properties of a stage-specific self-efficacy scale for physical activity using classical test theory, confirmatory factor analysis and item response modeling. Health Educ Res. 2006; 21(Supplement 1):i33-i46.

27. Sniehotta F, Scholz U, Schwarzer R. Bridging the intentionbehaviour gap: Planning, self-efficacy, and action control in the adoption and maintenance of physical exercise. Psychol Health. 2005; 20(2):143-60.

28. Morris K, McAuley E, Motl R. Self-efficacy and environmental correlates of physical activity among older women and women with multiple sclerosis. Health Educ Res. 2008; 23(4):744-52.

29. Scholz U, Sniehotta F, Schwarzer R. Predicting Physical Exercise in Cardiac Rehabilitation: The Role of PhaseSpecific Self-Efficacy Beliefs. J Sport \& Exerc Psychol. 
2005; 27:135-51.

30. Schwarzer R, Luszczynska A, Ziegelmann J, Scholz U, Lippke $S$. Social-Cognitive predictors of physical exercise adherence: Three longitudinal Studies in rehabilitation. Health Psychol. 2008; 27(1S):S54-63

31. Clark M, Abrams D, Niaura R, Eaton C, Rossi J. Self-Efficacy in Weight Management. J Consult Clin Psychol, 1991: 59(5):739-44.

32. Román Y, Díaz B, Cárdenas M, Lugli Z. Construction and validation of the perceived self-efficacy inventory for weight control. Clin Salud. 2007; 18 (1):45-56.

33. Glynn S, Ruderman A.The Development and Validation of an Eating Self-Efficacy Scale. Cog Therapy Res. 1986; 10(4):403-420.

34. Dutton G, Davis MP, Rhode P, Brantley P. Use of the Weight Efficacy Lifestyle Questionnaire with African American women: Validation and extension of previous findings. Eating Behav. 2004; 5:375-84.

35. Wilson-Barlow L, Hollins T, Clopton, J. Construction and validation of the healthy eating and weight self-efficacy (HEWSE) scale. Eating Behav. 2014; 15: 490-92.

36. Ruiz VM, Berrocal C, López AE, Rivas T. Self-efficacy in the control behavior intake. Adaptación al castellano de la Eating Self-Efficacy Scale. Psicothema. 2003; 15(1):36-40.

37. Sallis J, Pinski R, Grossman R, Patterson T, Nader P. The development of self-efficacy scales for health-related diet and exercise behaviors. Health Educ Res. 1988; 3(3):283-92.

38. Stich C, Knauper B, Tint A. A scenario-based dieting self-efficacy scale: The DIET-SE. Assessment. 2009; 16(1):16-30.

39. Stanton A, García M, Green S. Development and validation of the situational appetite measures. Addictive Behav. 1990; 15:461-72.

40. Stotland S, Zuroff D, Roy M. Situational Dieting SelfEfficacy and Short-Term Regulation of Eating. Appetite. 1991; 17;81-90.

41. Aedo A, Ávila H. New questionnaire to assess self-efficacy towards physical activity in children. Rev Panam Salud Pública. 2009; 26(4):324-29.

42. Guzmán SR, Gómez PG. Adaptation of the perceived selfefficacy inventory for weight control and physical activity for Mexican population. In: Rivera AS, Díaz-Loving $R$, Reyes LI, Sánchez, AR, Cruz M. (Eds.). La Psicología Social en México. México: AMEPSO, pp 475-83. 2010.

43. Guzmán SR, Gómez PG. Discriminant Analysis of the Self-efficacy Inventory for Weight Control in Mexican Adolescents. Psicol Salud. 2011; 21(2):157-64.

44. Guzmán SR, Gómez PG, García MM, del Castillo AA. Confirmatory factorial analysis of the perceived self-efficacy inventory for weight control in the Mexican population. Psicol Iberoam. 2011; 19(2):78-88.

45. Flores $L A$, González-Celis RA, Valencia OA. Validation of the Self-Efficacy scale for healthy behavior In healthy Mexican children. Psicol Salud. 2010; 20(1):23-30.
46. Gómez PG, León HR, Rodríguez SJ, Maza VX, Platas AS. Validation of the self-efficacy healthy behaviors inventory in Mexican school children. In: Rivera AS, Díaz-Loving $R$, Reyes LI, Flores GM. (Eds.). La Psicología Social en México. México: AMEPSO, pp 747-753, 2014.

47. Encuesta Nacional de Salud y Nutrición 2012. National Results. Cuernavaca, México: Instituto Nacional de Salud Pública, 2012.

48. Swithers S. Artificial sweeteners produce the counterintuitive effect of inducing metabolic derangements. Trends Endocrin Metab. 2013; 24(9):431-41.

49. Zaragoza MA, Norte NA, Fernández SJ, Hurtado SJ, Ortiz $M R$. Type of beverages consumed by university students. Rev Esp Nutr Com. 2013; 19(2):114-9.

50. Fagherazzi G, Vilier A, Saes SD, Lajous M, Balkau B, Clavel-Chapelon F. Consumption of artificially and sugarsweetened beverages and incident type 2 diabetes in the Etude Epidémiologique aupre's des femmes de la Mutuelle Générale de l'Education Nationale-European Prospective Investigation into Cancer and Nutrition cohort. Am J Clin Nutr. 2013; 97(3):517-23.

51. Byrne B. Structural equation modeling with AMOS. USA: Lawrence Erlbaum, 2001.

52. Martínez AR, Hernández LLM, Hernández LLV. Psicometría. Madrid: Alianza, 2006.

53. Pérez GJ, Chacón MS, Moreno RR. Construct validity: The use of exploratory-confirmatory factor analysis for obtain evidence of validity. Psicothema. 2000; 12(Supl. 2):442-6.

54. Díaz-Loving R. Contributions of Mexican ethnopsychology to the resolution of the etic- emic dilemma in personality. J Cross-Cult Psychol. 1998; 29(1):114-8.

55. Palacios DJ. Evaluation of Mexican personality traits. Segundo Coloquio de Psicología; Sept 27; Universidad Humanitas, México, 2014.

56. Palacios DJ. Psychometric properties of the seeking sensation inventory for adolescents in Mexico (IBS-MX). Int J Psychol Res. 2015; 8(1):46-60.

57. Reyes LI. Measure personality in Mexico. Rev Psicol Soc Pers. 1996; 12:31-59.

58. Palacios DJ, Bustos AM. Cognitive social theory to promote pro-environmental behavior: Constructs evaluation. In: Díaz-Loving $R$, Rivera AS, Reyes LI, Eds. La Psicología Social en México. México: AMEPSO, pp 12-17, 2012.

59. Palacios DJ, Bustos AM. The rol of theory in developing psycho-environmental interventions. Psych Interv 2012;21(3):245-57.

60. Maibach E, Murphy D. Self-efficacy in health promotion research and practice: conceptualization and measurement. Health Educ Res. 1995; 10(1):37-50.

61. Hair J, Anderson R, Tatham R, Black W. Multivariate Analysis. 5a Edición. Madrid: Prentice Hall, 1999.

62. Regulation of the General Law of health in the research for health. Rev Salud Pública Nutr. 2003; 4(3):768-76. 